\title{
Asimetría del Ventrículo Laríngeo. Un Estudio Anatómico Directo en Material Cadavérico
}

\author{
Asymmetry of Laryngeal Ventricle. A Direct Anatomical Study in Cadavers
}

Bladimir Saldarriaga Tellez*; Edgar Giovanni Corzo Gómez**; Oscar Javier Niño*** \& Pedro Luis Forero Porras****

SALdARRIAGA, T. B. ; CORZO, G. E. G.; NIÑO, O. J. \& FORERO, P. P. L. Asimetría del ventrículo laríngeo. Un estudio anatómico directo en material cadavérico. Int. J.Morphol., 34(3):1092-1096, 2016.

RESUMEN: Por su localización anatómica en el cuello, su papel funcional en la respiración en la fonación y su importancia en los procesos de intubación endotraqueal, la caracterización morfológica de la laringe reviste gran importancia en el campo de la morfología, fonoaudiología y de especialidades clínicas como la otorrinolaringología y anestesia. En una muestra de 16 laringes fijadas en formol del anfiteatro de la Universidad Autónoma de Bucaramanga, se registraron las longitudes de las estructuras cartilaginosas, comparando el lado derecho y el lado izquierdo. No se encontraron diferencias significativas en las estructuras cartilaginosas de la laringe de acuerdo al lado (asimetría), excepto en la amplitud del ventrículo laríngeo. Se midió la profundidad del ventrículo laríngeo en el punto medio; el ventrículo laríngeo derecho presentó una profundidad promedio de 2,50 mm. y el izquierdo de 2,31 mm, presentando diferencia significativa $(\mathrm{p}=0,0102)$. Las diferencias encontradas se deben a la forma que tienen los pliegues vestibulares y vocales; en unas muestras los pliegues son aplanados, lo que hace que la amplitud sea uniforme, en otras los pliegues tienen forma de $\mathrm{S}$ itálica, lo que ocasiona variaciones en la profundidad del ventrículo. Teniendo en cuenta que la amplitud del ventrículo laríngeo varía dependiendo del estado funcional de la laringe, ya sea durante la respiración, o la fonación, este hallazgo, representa un aporte importante para motivar la realización de estudios, con un tamaño de muestra más grande, en otros grupos poblacionales y estudios funcionales que determinen si las variaciones del espacio tienen efecto en la fonación.

PALABRAS CLAVE: Ventrículo laríngeo; Asimetría laríngea; Morfometría láringea.

\section{INTRODUCCIÓN}

Desde el punto de vista evolutivo, la laringe es el órgano que permite la protección de las vías respiratorias durante la deglución sin embargo da lugar al paso del aire desde la faringe hacia la tráquea. En el niño, la laringe presenta una ubicación más alta, inicia a nivel del cuerpo de la vértebra $\mathrm{C} 1$ y en el adulto a nivel del cuerpo de $\mathrm{C} 3$, a esto se le suma que en el niño la epiglotis es proporcionalmente más ancha (Monnier, 2011; Garrido et al., 2007). Su función fonatoria fue de posterior aparición y se basa en movimientos de las pliegues vocales a partir de cambios de presión generados durante la espiración. Estos movimientos son regulados por la contracción de músculos que actúan generando movimiento y tensión desde los cartílagos de la laringe hacia las pliegues vocales. Varios autores han descrito de forma directa, diferentes porcentajes de asimetría laríngea.
Joshi et al. (2011) estudiaron en 50 preparaciones de laringe de cadáveres adultos de la India la longitud de la laringe, longitud del pliegue ari-epiglótico, longitud de la pliegue vocal y del pliegue vestibular; encontraron un gran porcentaje de asimetría y datos que pueden aportar a la microcirugía de laringe y a la evaluación de los resultados de las técnicas diagnósticas. Zielinski (2001) estudió la importancia clínica de los diámetros de la laringe en cadáveres mayores de 65 años de ambos sexos. Shibusawa \& Yanok (1990) describieron en 315 personas japonesas la rotación de la laringe usando tomografía computarizada y señalaron como la rotación afecta la simetría de los pliegues vocales y su movilidad. Friedrich et al. (1988) estudió las asimetrías del cartílago tiroides y su relación con la región posterior de la glotis encontrando una correlación entre la configuración

\footnotetext{
* Profesor Facultad de Salud Universidad Autónoma de Bucaramanga, Universidad Industrial de Santander, Bucaramanga, Colombia.

** Profesor Facultad de Salud Universidad Autónoma de Bucaramanga, Bucaramanga, Colombia.

*** Programa de Medicina de la Universidad Autónoma de Bucaramanga, Bucaramanga, Colombia.

***** Profesor Asociado Universidad Industrial de Santander, Colombia. Instituto de Medicina Legal y Ciencias Forenses Regional Nororiente, Bucaramanga, Colombia.

Proyecto financiado en la Convocatoria interna de investigaciones Universidad Autónoma de Bucaramanga (UNAB) 2011-1012 Código C34036.
} 
supraglótica del cartílago tiroides y las anomalías de la glotis posterior; de igual manera señaló que el crecimiento irregular del cartílago tiroides causa variaciones de los pliegues ari-epiglóticos y de la ubicación de los tubérculos corniculados durante la fonación. Hirano et al. (1989) estudiaron en 50 laringes la asimetría tomando como punto de referencia para el marco laríngeo las láminas del cartílago tiroides; encontraron asimetría en mayor o menor medida en todas las laringes con inclinación hacia la dirección lateral de la lámina derecha del cartílago tiroides en tanto que la lámina izquierda tendía a una inclinación más medial. Frente a esta asimetría suponen que deben existir mecanismos de compensación del marco laríngeo asimétrico que puedan mantener una relativa simetría de las cuerdas vocales. Pereira Jotz et al. (2007) describen diferencias significativas de asimetría entre sexos, en el diámetro transverso anteroposterior del cartílago cricoides, pero no entre el diámetro interno del cartílago cricoides y el ángulo externo del cartílago tiroides, en población de Porto Alegre Brasil.

En Latinoamérica específicamente Venezuela, Morantes \& Narváez (2005), realizaron estudios para determinar la asimetría laríngea en función del eje glótico y parámetros funcionales, en profesionales de la voz. Reporon un predominio de pacientes con ejes glóticos desviados hacia la derecha $(68,5 \%)$, la cual se correlacionó con la presencia de disfonía. En referencia al ventrículo laríngeo, no encontramos publicaciones relacionadas con su caracterización morfológica o asimetría.

En el presente artículo se describieron las características morfométricas de algunas de las estructuras cartilaginosas y referentes anatómicos de la laringe en una muestra de 16 especímenes formolados que son parte del material de estudio del anfiteatro de la Universidad Autónoma de Bucaramanga, Colombia.

\section{MATERIAL Y MÉTODO}

El presente trabajo correspondió a un estudio no probabilístico y descriptivo transversal en el que se describieron las características morfométricas de la laringe en 16 muestras anatómicas, de laringe, tráquea y esófago, ubicadas en las instalaciones del anfiteatro de la Facultad de Ciencias de la salud de la Universidad Autónoma de Bucaramanga. No fue considerado el género, debido a la imposibilidad de determinarlo dado el procedimiento de entrega de estos tipos de órganos por parte del Instituto Nacional de Medicina Legal, Regional Nororiente. Las muestras se sometieron a fijación en solución de formol al $5 \%$ y posteriormente se realizó su disección retirando los músculos extrínsecos de la laringe para dejar visible el esqueleto cartilaginoso. Se excluyeron del estudio las muestras anatómicas que presentaron alteraciones morfológicas de la laringe debido a patologías del cuello, traumas o deformidades.

Las medidas se registraron con un calibrador digital Vernier Mitutoyo con las siguientes especificaciones: Super Caliper-Solar Powered- Series 500-No battery or origin reset needed for IP67 Digital Caliper. De resolución $0.01 \mathrm{~mm}$.

Los datos fueron digitalizados en Epiinfo 2002, y el análisis estadístico fue realizado con el software STATA 13. Se evaluó la normalidad de los datos, y para el análisis de las diferencias entre los grupos se utilizó la prueba $t$ de student. Los valores $p$ fueron considerados como significativos siendo menores a 0,05 (error alfa hasta de un 5\%). medidas:

En las muestra anatómicas se registran las siguientes

- Longitud de la laringe desde el borde superior de la epiglotis hasta el borde inferior del cartílago cricoides.

- Ancho de la lámina del cartílago cricoides (en su punto medio).

- Longitud del asta superior derecha del cartílago tiroides.

- Longitud del asta superior izquierda del cartílago tiroides.

- Profundidad de la incisura tiroidea superior.

- Ancho de la incisura tiroidea superior.

- Altura de la línea media anterior del cartílago tiroides.

- Altura en el punto medio anterior del cartílago cricoides.

- Altura de la membrana crico-tiroidea.

- Altura de la lámina del cartílago cricoides.

- Grosor de la lámina del cartílago cricoides.

- Longitud de la epiglotis.

- Longitud del pliegue vocal derecho.

- Longitud del pliegue vocal izquierdo.

- Altura del ventrículo laríngeo derecho.

- Altura del ventrículo laríngeo izquierdo

\section{RESULTADOS}

Se analizó la distribución de los datos en cada una de las variables de estudio mediante pruebas gráficas de normalidad y se encontró que las variables seguían una distribución aproximadamente normal, por lo que se determinó el promedio y desviación estándar como medida de tendencia central y dispersión respectivamente. (Tabla I).

Se midió la altura del ventrículo laríngeo, la longitud del pliegue vocal y la longitud del asta superior del cartílago tiroides de manera bilateral encontrando diferencia 
estadísticamente significativa (asimetría) únicamente entre la altura del ventrículo laríngeo derecho e izquierdo $(\mathrm{p}=$ 0.0102). En la Figura 1 se puede observar dicha diferencia en dos de los especímenes y en la Figura 2 se puede observar la diferencia de las medidas bilaterales.

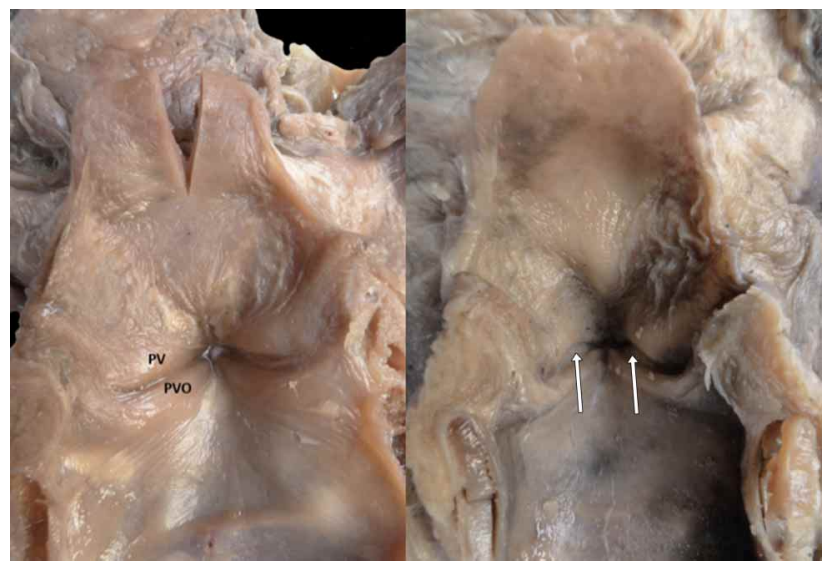

Fig. 1. Ventrículo laríngeo. A. Ventrículo laríngeo definido por pliegues vestibulares (PV) y vocales (PVO) planos con una leve diferencia en lado derecho e izquierdo. B. Ventrículo laríngeo delimitado por pliegues vestibulares y vocales en forma de $\mathrm{S}$ itálica. Las flechas señalan el ventrículo laríngeo.
De igual manera se observó en un espécimen una notoria asimetría ente las láminas del cartílago tiroides, con una notoria desviación de la lámina derecha del cartílago tiroides, con su consecuente efecto en la forma del ángulo tiroideo.

\section{DISCUSIÓN}

La revisión de la literatura evidencia la existencia de muy pocos trabajos de estudios poblacionales de las características morfométricas de la laringe a nivel mundial. Nuestros resultados están acordes a los reportados por Joshi et al. (2011), que describen las características de la laringe en población del oeste de la India encontrando una gran variabilidad en las longitudes de las estructuras de la laringe y en las distancias a referentes anatómicos; no encontraron diferencias significativas en la asimetría de las estructuras estudiadas. Se destaca la amplia variabilidad en la longitud de los pliegues vocales y vestibulares y diferencias en la simetría. Al comparar sus resultados con otros grupos poblacionales observan notables diferencias, lo que evidencia la existencia de variaciones relacionadas con el tamaño promedio corporal y de las características raciales.

Tabla I. Promedios de las mediciones morfométricas realizadas a cada una de las piezas.

\begin{tabular}{lcc}
\hline \multicolumn{1}{c}{ MEDIDA } & PROMEDIO $(\mathrm{mm})$ & DESVIACIÓN ESTÁNDAR \\
\hline Longitud de la laringe & 64,37 & 1,11 \\
Ancho de la lámina del cartílago cricoides & 24,93 & 0,83 \\
Longitud del asta superior derecha del cartílago tiroides & 16,62 & 1,48 \\
Longitud del asta superior izquierda del cartílago tiroides & 15,87 & 1,40 \\
Pro fundidad de la incisura tiroidea superior & 11,00 & 0,62 \\
Ancho de la incisura tiroidea superior & 13,68 & 1,11 \\
Altura de la línea media anterior del cartílago tiroides & 17,00 & 0,64 \\
Altura en el punto medio anterior del cartílago cricoides & 7,06 & 0,53 \\
Altura de la membrana cricotiroidea & 7,37 & 0,96 \\
Altura de la lámina del cartílago cricoides & 24,87 & 1,30 \\
Grosor de la lámina del cartílago cricoides & 4,50 & 0,40 \\
Longitud de la epiglotis & 37,43 & 1,07 \\
Longitud del pliegue vocal derecho & 14,37 & 0,80 \\
Longitud del pliegue vocal izquier do & 14,56 & 0,79 \\
Altura del ventrículo laríngeo derecho & 2,50 & 0,40 \\
Altura del ventrículo laríngeo izquierdo & 2,31 & 0,38
\end{tabular}



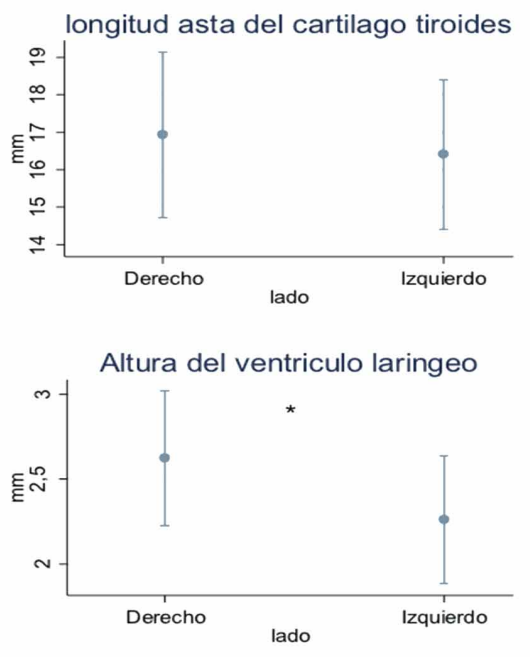

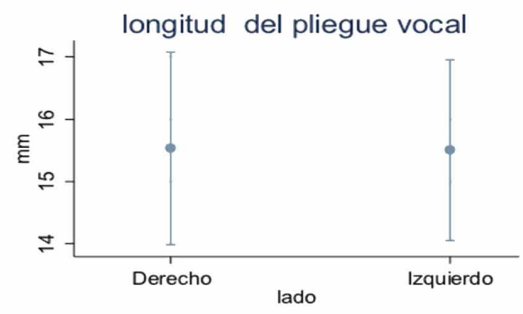

${ }^{*} p<0.05$ en prueba de diferencia de medias
Fig. 2. Comparación de las medidas bilaterales.
Jain (2008) reportó, en una muestra poblacional de Haryana (India) diferencias morfológicas de acuerdo al sexo y mayores longitudes para todos los parámetros medidos en individuos de sexo masculino, excepto para el ángulo tiroideo y la longitud del asta inferior del cartílago tiroides. La lámina tiroidea fue más oblicua con respecto al plano horizontal en mujeres comparadas con hombres, se encontró una correlación significativa entre el peso del individuo y la longitud de la lámina del cartílago tiroideo. En un $25 \%$ de los casos la prominencia laríngea no estaba localizada en la línea media, si no desviada hacia la derecha o hacia la izquierda. En nuestro estudio encontramos esta característica, solo en un espécimen.

Kova \& Popovic (2010) estudiaron las características morfométricas de la laringe en un grupo poblacional del este de Croacia encontrando diferencias significativas en todos los parámetros anatómicos de la laringe entre hombre y mujeres excepto para la distancia entre los extremos del asta superior del cartílago tiroides. En general todos los valores son mayores en el sexo masculino, excepto el ángulo del cartílago tiroides, que fue significativamente mayor en mujeres. Estos investigadores compararon sus resultados con los reportados en poblaciones de Alemania, Estados Unidos, India y Nigeria y encuentran concordancia en las diferencias anatómicas entre hombres y mujeres. Se encontraron notorias diferencias en los estudios en la longitud de las astas superiores e inferiores del cartílago tiroides, como también en las láminas del cartílago tiroides.

Zielinski (2001) estudió 52 laringes de personas entre los 65 y 81 años, 28 masculinas y 24 femeninas, registró cambios en los diámetros de la laringe medidos a diferentes niveles, su estudio sugiere que el proceso de envejecimiento produce cambios que son de importancia funcional y clínica.
La falta de asimetría en la mayor parte de los referentes anatómicos de la laringe encontrados en este trabajo, puede ser consecuencia del tamaño de la muestra objeto del estudio, o una característica propia de la población colombiana, lo que amerita la ampliación de estudios en otros grupos poblacionales. Las diferencias significativas encontradas en la amplitud del ventrículo laríngeo, se debe a las características anatómicas de los pliegues vocales y pliegues vestibulares. Este hallazgo anatómico puede ser de importancia, considerando que la amplitud del ventrículo laríngeo varía notoriamente durante la fonación. Dado que los pliegues vocales forman el piso del ventrículo laríngeo, su longitud y características pueden ser determinantes en la amplitud del ventrículo laríngeo. Se ha reportado que el tamaño del ventrículo laríngeo es mayor en cantantes y personas con buena voz, que en personas con pobre calidad vocal, lo que evidencia que el tamaño de ventrículo laríngeo puede tener una influencia en la acústica de la voz humana, su altura durante la fonación es de 3.0 mm. (Kitzing \& Sonesson 1967). En las muestras anatómicas estudiadas el promedio de la altura del ventrículo laríngeo derecho fue de $2,50 \mathrm{~mm}$ y $2.31 \mathrm{~mm}$. en el lado izquierdo.

La asimetría encontrada en la profundidad del ventrículo laríngeo ventrículo laríngeo es producida por las diferencias en la morfología de los pliegues vestibulares y pliegues vocales, estos presentaron forma aplanada lo que determina una profundidad uniforme de la amplitud del ventrículo laríngeo y en forma de S itálica; esta forma se presentó tanto en el lado derecho como en el lado izquierdo y en ambos pliegues y determina una mayor amplitud del ventrículo laríngeo (Fig.1A y 1B). La longitud promedio de los pliegues vocales derecho es de $14.57 \mathrm{~mm}$ y $14.36 \mathrm{~mm}$ el izquierdo.

AGRADECIMIENTOS. Agradecemos a la Universidad Autónoma de Bucaramanga por su apoyo y financiación al 
proyecto, desde la Dirección de Investigaciones, dentro del marco de convocatoria interna de investigaciones 2011-2012. Al Instituto Nacional de Medicina Legal Regional Nororiente por la entrega de las muestras anatómicas. Al Señor Carlos
Bermúdez Barajas por su apoyo en las disecciones y registros fotográficos; al doctor Sergio Eduardo Serrano Gómez, medico epidemiólogo vinculado a la UNAB por el procesamiento estadístico de los datos obtenidos en el trabajo.

SALDARRIAGA, T. B. ; CORZO, G. E. G.; NIÑO, O. J. \& FORERO, P. P. L. Asymmetry of laryngeal ventricle. A direct anatomical study in cadavers. Int. J.Morphol., 34(3):1092-1096, 2016.

SUMMARY: For its anatomical location in the neck, its functional role in breathing in phonation and its importance in the process of endotracheal intubation, the morphological characterization of the larynx is of great importance in the field of morphology, speech therapy and clinical specialties such as otolaryngology and anesthesia. In a sample of 16 larynges fixed in formalin at the amphitheater of the Universidad Autónoma de Bucaramanga., the lengths of the cartilaginous structures were recorded, comparing the right side and the left side. No significant differences in the cartilaginous structures of the larynx in reference to side (asymmetry) was found except amplitude of laryngeal ventricle. The depth of the laryngeal ventricle at the midpoint was measured; the right laryngeal ventricle showed an average depth of $2.50 \mathrm{~mm}$. and left $2.31 \mathrm{~mm}$, showing significant difference $(\mathrm{p}=0.0102)$. The differences are due to the vestibular and vocal folds; samples in the folds are flattened, making the amplitude uniform, in other folds they are shaped italic $\mathrm{S}$, causing variations in the depth of the ventricle. Given that the amplitude of the laryngeal ventricle varies depending on the functional state of the larynx, either during respiration or phonation, this finding represents an important contribution to motivate studies, with larger size sample, in other population groups and functional studies to determine whether variations in space have an effect on phonation.

KEY WORDS: Laringeal ventricle ; Laryngeal assymetry; Laryngeal morphometry.

\section{REFERENCIAS BIBLIOGRÁFICAS}

Friedrich, G.; Kainz, J. \& Anderhuber, F. The effect of the configuration of the thyroid cartilage on the asymmetry of the dorsal glottis and its significance for vocal function. H. N. O., 36(6):241-50, 1988.

Garrido, G. C.; Flores, H. S. S. \& Núñez Pérez-Redondo, C. Diferencias anatomofuncionales y endoscópicas entre la vía aérea del niño y la del adulto. Rev. Inst. Nac. Enferm. Respir., 20(2):142-8, 2007.

Hirano, M.; Kurita, S.; Yukizane, K. \& Hibi, S. Asymmetry of the laryngeal framework: a morphologic study of cadaver larynges. Ann. Otol. Rhinol. Laryngol., 98(2):135-40, 1989.

Jain, M. \& Dhall, U. Morphometry of the thyroid and cricoid cartilages in adults. J. Anat. Soc. India, 57(2):119-23, 2008.

Joshi, M. M.; Joshi, S. S. \& Joshi, S. D. The morphological study of adult human larynx in a Western Indian population. J. Laryngol. Voice, 1(2):50-4, 2011.

Kitzing, P. \& Sonesson, B. Shape and shift of the laryngeal ventricle during phonation. Acta Otolaryngol., 63(5):479-88, 1967

Kovac, T.; Popovic', B.; Marjanovic', K.; Wertheimer, V.; Kovacevic', M.; Nikolic', V.; Jo-Osvatic', A. \& Radic', R. Morphometric characteristics of thyroid cartilage in people of Eastern Croatia. Coll. Antropol., 34(3):1069-73, 2010.

Monnier, P. (Ed). Pediatric Airway Surgery. Management of Laryngotracheal Stenosis in Infants and Children. Heidelberg, Springer Verlag, 2011.
Morantes, L. \& Narvaez, U. Relación entre asimetría laringea, studio electromiográfico y análisis acústico en profesionales de la voz. Bol. Med. Postgrado, 21(3):1-10, 2005.

Pereira Jotz, G.; Zaquia Leao, H.; Pereira Da Costa Filho, O.; Fisch, P.; Coelho Magalhaes, R. \& Cervantes, O. The asymmetry index of the cricoid cartilage and the external angle of the thyroid cartilage. A sex-related study. Eur. J. Anat., 11(1):1-7, 2007.

Shibusawa, M. \& Yano, K. Deviation and rotation of the larynx in computer tomography. Nihon Jibiinkoka Gakkai Kaiho, 93(1):15,1990 .

Zielinski, R. Morphometrical study on senile larynx. Folia Morphol. (Warsz.), 60(2):73-8, 2001.
Dirección para correspondencia:
Profesor Bladimir Saldarriaga Téllez
Universidad Autónoma de Bucaramanga
Calle 157 \# 14 -55 Cañaveral-Parque
Floridablanca-Santander
COLOMBIA

Email: vsaldarr@unab.edu.co

Recibido : 14-07-2015

Aceptado: 05-05-2016 